Published in final edited form as:

Perspect ASHA Spec Interest Groups. 2016 July ; 1(2): 82-95. doi:10.1044/persp1.SIG2.82.

\title{
Acute Ischemic Lesions Associated with Impairments in Expression and Recognition of Affective Prosody
}

\author{
Amy E. Wright, \\ Department of Neurology, Johns Hopkins University, School of Medicine, Baltimore, MD \\ Cameron Davis, \\ Department of Neurology, Johns Hopkins University, School of Medicine, Baltimore, MD \\ Yessenia Gomez, \\ Department of Neurology, Johns Hopkins University, School of Medicine, Baltimore, MD \\ Joseph Posner, \\ Department of Neurology, Johns Hopkins University, School of Medicine, Baltimore, MD \\ Christopher Rorden, \\ Center for Aphasia Research and Rehabilitation, University of South Carolina, Columbia, SC
}

Argye E. Hillis, and

Department of Neurology, Johns Hopkins University, School of Medicine, Baltimore, MD.

Department of Physical Medicine and Rehabilitation, Johns Hopkins University School of

Medicine, Baltimore, MD. Department of Cognitive Science, Krieger School of Arts and Sciences,

Johns Hopkins University, Baltimore, MD

\section{Donna C. Tippett}

Department of Neurology, Johns Hopkins University, School of Medicine, Baltimore, MD. Department of Physical Medicine and Rehabilitation, Johns Hopkins University School of Medicine, Baltimore, MD. Department of Otolaryngology—Head and Neck Surgery, Johns Hopkins University School of Medicine, Baltimore, MD

\section{Abstract}

Purpose-We aimed to: (a) review existing data on the neural basis of affective prosody;(b) test the hypothesis that there are double dissociations in impairments of expression and recognition of affective prosody; and (c) identify areas of infarct associated with impaired expression and/or recognition of affective prosody after acute right hemisphere (RH) ischemic stroke.

Methods-Participants were tested on recognition of emotional prosody in content-neutral sentences. Expression was evaluated by measuring variability in fundamental frequency. Voxelbased symptom mapping was used to identify areas associated with severity of expressive deficits.

Disclosures

Financial: The research reported in this paper was supported by the National Institutes of Health (National Institute of Neurological Disorders and Stroke) through award R01 NS04769 and P41 EB015909. The content is solely the responsibility of the authors and does not necessarily represent the views the National Institutes of Health.

Nonfinancial: The authors have no relevant nonfinancial information to disclose. 
Results-We found that 9/23 patients had expressive prosody impairments; $5 / 9$ of these patients also had impaired recognition of affective prosody; $2 / 9$ had selective deficits in expressive prosody; recognition was not tested in $2 / 9$. Another $6 / 23$ patients had selective impairment in recognition of affective prosody. Severity of expressive deficits was associated with lesions in right temporal pole; patients with temporal pole lesions had deficits in expression and recognition.

Conclusions-Expression and recognition of prosody can be selectively impaired. Damage to right anterior temporal pole is associated with impairment of both, indicating a role of this structure in a mechanism shared by expression and production of affective prosody.

\section{The Role of Expression and Recognition of Affective Prosody in Communication}

Accurate and effective verbal communication requires more than just syntax and semantics; we rely on far subtler components to infuse meaning and emotional information into spoken messages. These components, collectively known as prosody, include rate, pitch, and loudness of speech, as well as the placement of stress on words and syllables. The ability to recognize affective intention is vital for accurate perception of a communication partner's emotional state andgeneration of an appropriate response to this circumstance. Conversely, the ability to convey one's own emotional information and intention through speech effectively is critical to successful communication between partners as well (Nöth, Batliner, Kießling, Kompe, \& Niemann, 2000). There are several different types of prosody. For example, linguistic or intrinsic prosody differentiates declarative, interrogative, imperative, and exclamatory sentences, and disambiguates wordsand phrases (Wong, 2002). Lexical or phonemic stress differentiates words, such as "project"(i.e., assignment) as opposed to "project" (i.e., propel). Contrastive stress, or word emphasis(Cruttenden, 1997), clarifies meaning, distinguishing between the phrase "I want cereal for breakfast" (I, not others, want cereal) as compared to "I want cereal for breakfast" (I want cereal, not something else, for breakfast). Intellectual prosody conveys attitudinal information, such as distinguishing sincerity (e.g., "The weather is lovely today" spoken with emphasis on "is") versus sarcasm (e.g., "The weather is lovely today" spoken with emphasis on "lovely" and rising terminal intonation). Emotional prosody reveals the sentiment of the speaker, such as conveying sadness via a quiet voice with a falling pitch contour, anger via a loud, harsh voice and fast rate of speech. Affective or extrinsic prosody, the focus of our study, includes both intellectual and emotional prosody (Ross, 2000).

\section{Deficits in Affective Prosody After Stroke}

Survivors of right hemisphere (RH) stroke may have deficits in recognition or expression of prosody, or a combination of both. An individual with expressive deficits may have a flat, monotone pattern of speech that does not convey emotional connotation (Tucker, Watson, \& Heilman, 1977). An individual with impaired recognition of affective prosody may be able to understand the overt, propositional content of a message, but may be unable to recognize the emotion conveyed (Bowers, Coslett, Bauer, Speedie, \& Heilman, 1987; Weintraub, Mesulam, \& Kramer, 1981). Affective prosody impairments represent a subset of the communication impairments that can result from RH stroke. 
Even though 50-78\% of RH stroke survivors have one or more deficits in communication (Benton \& Bryan, 1996; Blake, Duffy, Myers, \& Tompkins, 2002; Côté, Payer, Giroux, \& Joanette,2007), the lesions within the RH responsible for the various communication deficits have not been well characterized. In contrast, there have been many investigations of lesions responsible for various forms of hemispatial neglect after RH stroke (Samuelsson, Jensen, Ekholm, Naver,\& Blomstrand, 1997; Vallar, 2001). Although hemispatial neglect is the deficit that most readily comes to mind (to most clinicians) as a cognitive sequel of $\mathrm{RH}$ damage, our recent work has shown that impairment in affective prosody (recognizing and expressing emotion in tone of voice) is a much more common consequence of acute $\mathrm{RH}$ ischemic stroke (Dara, Bang, Gottesman, \& Hillis, 2014). Not only is acute RH dysfunction better predicted by emotional prosody impairments as compared to neglect (Dara et al., 2014), it can also lead to important breakdowns in communication and relationships. Interpretation and use of prosodic elements are crucial for communicative interactions and for the well-being of speakers and communication partners (Carton, Kessler, \& Pape, 1999; Heilman, 2002; Ross, 1997; Trauner, Ballantyne, Friedland, \& Chase, 1996; Voeller, 1995; Weintraub \& Mesulam, 1983; Wymer, Lindman, \& Booksh, 2002). Recognition of prosody is one means to judge emotional tenor, and thus evoke an appropriately empathetic response. Failure to recognize the emotional state of another can result in failed interpersonal interactions. In a study of 12 individuals with RH stroke, ability to decode the meaning of facial expression and prosody was associated with decreased marital satisfaction (Blonder, Pettigrew, \& Kryscio, 2012). Furthermore, impaired prosody can be mistaken for depression, apathy, or impaired emotional empathy (making inferences about and sharing in emotions of others). Repeated problems in recognition or expression of affective prosody, leading to failures of communication of intent, can impede social roles (Roth et al., 2011). In one recent study, impaired recognition of emotions of others was the most common, important residual consequence of stroke reported among caregivers of RH stroke survivors (Hillis \& Tippett, 2014). Thus, impairments in affective prosody are both common and clinically important. It would be useful to identify specific lesion sites responsible for these deficits, and to better characterize the cognitive mechanisms underlying these important functions. In this paper, we first review previous studies of the neural basis of affective prosody. We then present new data from patients with acute ischemic stroke on lesions that produce impairments in expression and/or recognition of affective prosody.

\section{Hemispheric Dominance for Prosody}

Right hemisphere (RH) specialization for perception and production of emotion has long been recognized (Blonder, Bowers, \& Heilman, 1991; Borod, 2000). Hughlings Jackson (1874) hypothesized that the RH plays a unique role in the emotional aspects of communication, an idea supported by his observations that even those with pronounced aphasia are able to convey messages via modulation of speech output. The dominant role of the $\mathrm{RH}$ in processing emotional prosody is also supported by studies recording event-related brain potentials (Pihan, Altenmuller, Hertrich, \& Ackermann, 2000); functional magnetic resonance imaging (fMRI) studies showing RH activation in association with prosody judgments (Buchanan et al., 2000); and lesion studies of judging emotional meaning from 
prosody (Blonder et al., 1991; Cancelliere \& Kertesz, 1990; Pell, 2006; Ross \& Monnot, 2008).

Linguistic prosody is modulated by both the left hemisphere (LH) and RH (Balan \& Gandour, 1999; Baum, Pell, Leonard, \& Gordon, 1997; Gandour \& Baum, 2001; Heilman, Bowers, Speedie, \& Coslett, 1984; Schirmer, Alter, Kotz, \& Friederici, 2001; Walker, Joseph, \& Goodman, 2009; Walker, Pelletier, \& Reif, 2004). However, LH damage is unique from RH damage in part because it can cause particular difficulty modulating temporal parameters and inconsistent difficulty modulating frequency related parameters (Shah, Baum, \& Dwivedi, 2006; Wong, 2002). Theoretical explanations for hemispheric specialization include the "task-dependent" or "functional" theory and the "cue-dependent" or "acoustic" theory. According to the "task-dependent" theory, more linguistic type cues lateralize to the $\mathrm{LH}$, and more affective-emotional type cues lateralize to the RH (Van Lancker, 1980). The "cue-dependent" theory posits that lateralization of prosody is based on acoustic parameters. If the cue is signaled by temporal features, then it lateralizes to the LH. If the cue is signaled by spectral features, then it lateralizes to the RH (Robin, Tranel, \& Damasio, 1990; Van Lancker \& Sidtis, 1992). A recent investigation of the neuroacoustic basis of prosodic stress in individuals with left and right brain damage supports the "taskdependent" theory rather than the "cue-dependent" theory (Ross, Shayya, \& Rousseau, 2013).

\section{Specific Lesions Associated With Expression Versus Recognition of Affective Prosody}

Early lesion studies by Gorelick and Ross (1987) and Ross and Mesulam (1979) supported the notion that the functional-anatomic organization of prosody in the RH mirrors that of propositional language in the LH. For example, loss of the ability to modulate affective prosody was found to be associated with lesions in right frontal operculum, a homolog of Broca's area. Loss of ability to comprehend affective prosody was found to be associated with lesions in right temporal operculum, a homolog of Wernicke's area (Ross, 2010). Subsequent studies, however, have not confirmed these associations. For example, Breitenstein, Daum, and Ackermann (1998) found similar comprehension deficits in patients with anterior, as opposed to posterior, RH lesions. However, of the 16 participants only 4 had strokes, with the other 12 participants' lesions of various etiologies including gunshot wound and brain tumor excision. Slow-growing tumors notoriously result in gradual reorganization of structure-function relationships, such that the tumor or resection often does not produce deficits even if it occurs in an area normally critical for important functions.

Inconsistencies in imaging acquisition and analysis represent other possible explanations for the contradicting reports from previous studies. One study reported that $80 \%(16 / 20)$ of patients with RH stroke were classified by Ross's aprosodia taxonomy (e.g., global aprosodia, motor aprosodia, sensory aprosodia); four individuals were unclassifiable. The aprosodia types, however, did not systematically relate to lesion site. For example, some individuals with motor aprosodia did not have lesions in the RH analogous to LH lesions that cause Broca's aphasia (Wertz, Henschel, Auther, Ashford, \& Kirshner, 1998). This 
study had a heterogeneous participant pool, with 5/20 participants having hemorrhagic strokes, and only 16/20 receiving a computed tomography (CT) scan or MRI. But most notably there was no formal lesion mapping done, which raises the question of the validity of the relationship (or lack thereof) between prosody classification and lesion location. Additionally, there was no indication of when patients were scanned in relation to their testing (for those who were scanned). Some studies have also shown that deficits in affective prosody production and comprehension can occur due to damage to the basal ganglia and thalamus (Breitenstein et al., 1998; Cohen, Riccio, \& Flannery, 1994; Karow \& Connors, 2003; Sidtis, Pachana, Cummings, \& Sidtis, 2006; Wolfe \& Ross, 1987), or white matter tracts (Bowers, Bauer, \& Heilman, 1993), which are areas that were not investigated in previous studies.

The early studies by Ross, Heilman, and others reported dissociations in recognitionand expression of affective prosody, indicating that these can be independently impaired by brain damage (Bowers et al., 1993; Ross \& Monnot, 2008). However, most studies of lesions associated with impaired prosody have been conducted in individuals with fairly chronic lesions, after potential recovery and reorganization of structure-function relationships. It is possible that both were impaired initially, and one aspect of prosody (e.g., recognition) recovered more quickly in some individuals. It may be that intact areas of the brain may be able to assume recognition or expression of prosody more readily. One way to address this limitation is to study patients acutely after stroke, before the opportunity for recovery and before other areas of the brain assume the function of the damaged areas. This approach would allow us to identify areas responsible for these critical functions before reorganization.

Therefore, this study aimed to: (a) test the hypothesis that there are double dissociations in impairments of expression and recognition of affective prosody in acute $\mathrm{RH}$ ischemic stroke; and (b) identify specific areas of acute infarct associated with impaired expression and/or recognition of affective prosody.

\section{Method}

\section{Participants}

Twenty-three patients with acute RH ischemic strokes were admitted to the stroke service at Johns Hopkins Hospital were recruited and enrolled in the study. Participants were aged 3972 years $(M=57.78, S D=9.90)$, with a mean education level of $13.0 \pm 3.1$ years, and included 8 females. Participants provided informed consent and met the following inclusion criteria: premorbid proficiency in English; ability to understand tasks and follow commands; no known hearing loss or uncorrected visual impairment; no history of prior symptomatic stroke, dementia, or other neurological disease; and no hemorrhage present on first clinical scan. Patients were excluded if they were unable to have MRI (e.g., due to claustrophobia, implanted ferrous metal, or weight $>300 \mathrm{lb}$.), had reduced level of consciousness, or ongoing sedation. 


\section{Prosody Testing}

Within four days of stroke symptom onset, patients were administered two of the five subtests of the Aprosodia Battery (Ross, Thompson, \& Yenkosky, 1997). The Aprosodia Battery (Ross et al., 1997) assesses comprehension and production (spontaneous and repetition) of affective prosody and quantifies results to distinguish patterns of deficits. Patients evaluate stimuli to assess if the same or different emotions are expressed on a discrimination task. Patients also engage in repetition and pontaneous speech tasks and speech output is recorded and then analyzed acoustically. The battery is especially sensitive to distinguishing between deficits stemming from right versus left cortical hemisphere damage, with the verbal-articulatory demands reduced incrementally as each subtest progresses. These tasks have been used to study several different clinical populations with robust results, including patients with Alzheimer's disease (Testa, Beatty, Gleason, Orbelo, \& Ross, 2001), multiple sclerosis (Beatty, Orbelo, Sorocco, \& Ross, 2003), posttraumatic stress disorder (Freeman, Hart, Kimbrell, \& Ross, 2009), schizophrenia (Ross et al., 2001), and normal aging (Orbelo, Grim, Talbott, \& Ross, 2005).

In our study, two subtests were administered: Production/Expression (Repetition) and Recognition (Identification). Both subtests used the same sets of stimuli. There were three tasks in each subtest, the titles of which reflect the type of utterances presented: Word ("I am going to the other movies"); Monosyllabic ("ba ba ba ba ba ba"); and Asyllabic ("aaaaaaahhh"). Each task is comprised of 12 content-neutral utterances carrying six emotional tones (happy, sad, angry, surprised, disinterested, and neutral) repeated twice: one with the emphatic stress early in the utterance and once with the stress near the end of the utterance. Stimuli were presented using the software package Presentation (Neurobehavioral Systems, Albany, CA).

To assess Production, participants completed three repetition tasks (word, monosyllabic, and asyllabic). Participants were instructed to listen to the utterances and repeat them aloud using the same tone of voice as that modeled by the speaker. Each of the three tasks was preceded by four practice trials to ensure the participants' comprehension of the task. Participants' utterances were recorded in.wav format using Tascam DR-100 Portable Digital Recorder, and analyzed using Praat (n.d.), a speech analysis software. Praat was used to isolate each individual utterance, extract the selected sounds, and convert them to fundamental frequency (F0) using a filter of $350 \mathrm{~Hz}$. The mean and standard deviation of each individual utterance were obtained using the "Query" function in Praat and used to calculate the coefficient of variation of fundamental frequency for each utterance (F0-CV\%; standard deviation divided by mean F0, converted to percent). The F0-CV\% values were averaged across all 12 utterances of each task, resulting in three mean $\mathrm{F} 0-\mathrm{CV} \%$ values for each patient. The three mean F0-CV\% values of each patient were subsequently compared to the corresponding values of normal controls as reported by Ross and Monnot (2008), with patients' age 40-59 years comprising the younger group and patients age 60-79 years comprising the older group. For the purpose of this study, mean F0-CV\% scores falling one or more standard deviations below the appropriate age group's normative values were considered abnormal. 
To assess comprehension, participants were tested using the three Identification tasks. Each of the three tasks (Word Identification, Monosyllabic Identification, and Asyllabic Identification) comprised of the same 12 utterances used in the Repetition tasks, including two renditions of each of the six emotional tones under investigation: happy, sad neutral, angry, surprised, and disinterested. Participants were trained to depress a button labeled with the printed names of the six emotional tones. Before the start of testing, each participant demonstrated the ability to read the labels and comprehend the task directions. Participants were instructed to identify the emotional tone of the speaker after listening to each utterance by depressing the correct button. Responses were automatically recorded using Presentation (Neurobehavioral Systems, Albany, CA).

\section{Imaging}

Within 48 hours of behavioral testing, patients underwent magnetic resonance (MR) scanning, including diffusion weighted imaging (DWI). Lesion maps were manually drawn on the mean diffusivity image generated from the DWI sequence. The un-weighted (B0) image from this sequence was used to estimate spatial normalization. This approach exploits the fact that the acute lesion is readily identified on the Mean Diffusivity (MD) image, yet its absence on the B0 image ensures that the injury does not disrupt normalization (Mah, Jager, Kennard, Husain, \& Nachev, 2014). Statistical Parametric Mapping (SPM8), software for analyzing brain imaging data, was used for spatial processing. The location of the anterior commissure was estimated by initially finding the center of brightness for the volume followed by followed by coregistration to the echo-planar imaging (EPI) template, a representative image with anatomical features in a coordinate space to which individual images can be aligned. Subsequently, the images were normalized to the EPI template. Both the B0 image and lesion maps were resliced to standard space using these transforms. These resliced lesions were converted back to binary maps using a threshold of 0.5 . A population mean B0 image was generated to check the quality of normalization and as a background image for the statistical maps. Lesion subtraction maps (Rorden \& Karnath, 2004) were generated to highlight regions typically injured in impaired regions and spared in individuals without deficits. For lesion symptom mapping, permutation thresholded t-tests, using mean $\mathrm{Z}$-score of the production tasks were computed as the index of impairment.

\section{Results}

Nine of the 23 patients had expressive prosody impairments, indicated by a mean F0-CV\% of at least one standard deviation below the means for their age and gender. Of these nine patients, five also had impaired prosody recognition; two had selective deficits in expressive prosody; and two did not complete the prosody recognition tasks. Six other patients had selective impairment in recognition of prosody.

Lesion symptom mapping was carried out only for expressive prosody impairment because there was a sufficient number of patients with adequate variation in severity only for expressive deficits. For expressive affective prosody impairment, right temporal pole survived a one-tailed $\mathrm{p}<0.05$ (threshold Z-score $<-2.45$ ) with an observed Z-score of -2.46 (see Figure 1). All patients with right temporal pole lesions had deficits in both 
expression and recognition of affective prosody. The two patients with selective deficits in expressive prosody had right frontal lesions. Patients with selective deficits in receptive prosody had posterior temporal $(n=3)$, caudate $(n=2)$, or thalamic $(n=1)$ lesions.

\section{Discussion}

A double dissociation between impaired modulation of expressive prosody (with normal recognition of affective prosody) in some cases and impaired recognition of affective prosody (with intact expressive prosody) in other cases after acute stroke, provides evidence that there are at least some distinct mechanisms underlying expression and recognition of prosody that can be selectively impaired even by acute lesions. The two patients with selective deficits in expressive prosody had right frontal lesions, while the patients with selective deficits in recognition of affective prosody had caudate, thalamic, or posterior temporal lesions. These results are consistent with findings from Ross and Monnot (2008) indicating that the right posterior-inferior frontal region plays a critical role in affective prosody production, and the right posterior-superior temporal region is essential for affective prosody recognition. These results are also consistent with the proposal that the affective prosody network "mirrors" the language network of the LH. It is widely (although not universally) agreed that the left posterior-inferior frontal region is necessary for speech production, while the left posterior-superior temporal lobe is important in verbal-semantic comprehension (Hillis et al., 2001; Hillis et al., 2004; Kreisler et al., 2000).

Patients with impairment in both expression and recognition of affective prosody had lesions mostly involving right anterior temporal pole, and this area was statistically associated with severity of their expressive deficit. This region may be critical for a component common to expression and recognition of prosody, such as the semantic representation of the emotion to be conveyed. These results are consistent with previous reports that the anterior temporal poles (bilaterally) function as a "semantic hub" in object meaning (Pobric, Jefferies, \& Lambon Ralph, 2010) and have been proposed as a critical for understanding social concepts (Zahn et al., 2009). Our results indicate a role of this structure in a mechanism shared by expression and production of affective prosody, such as representing the meaning of emotions.

The role of right temporal pole in both expression and recognition of affective prosody is also supported by another recent study from our laboratory (unpublished data). We evaluated 35 patients with RH stroke, 50 patients with LH stroke, and 18 age-matched controls, using Praat to evaluate the variation in expressive prosody (measured by mean $\mathrm{F} 0-\mathrm{CV} \%$ ) while participants described a picture as part of the Boston Diagnostic Aphasia Examination (Goodglass \& Kaplan, 1983). The single patient with an abnormally low F0-CV\% in the picture description task had hypoperfusion of the right temporal pole as well as both impaired expressive prosody and impaired comprehension of prosody (see Figure 2). The remaining 84 patients had $\mathrm{F} 0-\mathrm{CV} \%$ which fell within the range of normal.

Finally, additional support for the critical role of right temporal pole in affective prosody expression and recognition was reported by Dara et al. (2014). They reported the performance of a patient with behavioral variant frontotemporal dementia who performed 
normally on most neuropsychological tests, but performed remarkably poorly on several prosody tasks. In particular, this patient demonstrated severely impaired recognition of affective prosody (tested on the Aprosodia Battery), as well as severely reduced variation in prosody in emotional tone in repetition and in describing emotional events (measured with the $\mathrm{F} 0-\mathrm{CV} \%$ ), associated with severe atrophy in right temporal pole (see Figure 3). Henry et al. (2014) reported a similar case with progressive social-emotional and semantic deficits associated with predominantly right anterior temporal atrophy.

We propose an architecture of the cognitive processes underlying affective prosody, adapted from proposals by Bowers et al. (1993). Affective prosody recognition begins with auditory analysis of the stimulus, which allows: (a) identification of the prosodic features: mean and variation in frequency, amplitude, and rate of speech, which together allows (b) access to the "affective prosody lexicon" (representations that specify the set of speech dimensions corresponding to an emotion (e.g., <low volume $><$ slow rate $><$ reduced pitch variation $>$ for "sad"), which allows (c) access to the semantic representation (meaning) of $\langle$ sad $\rangle$ (see Figure 4). Affective prosody production begins with the semantic representation, which allows access to the affective prosody lexicon (for output), then motor programming of cricothyroid, cricoarytenoid, and vocalis muscles and muscles of articulation and respiration needed to produce changes in pitch, loudness, and rate of speech.

Impaired receptive and expressive prosody have been reported in patients with different lesions. In this study, five patients with selective deficits in prosody recognition had posterior temporal $(n=3)$, caudate $(n=2)$, or thalamic $(n=1)$ lesions. We did not perform adequate testing to distinguish whether their deficits were in identification of the prosodic features (auditory analysis of mean and variation in frequency, amplitude, and rate of speech) or in access to the "affective prosody lexicon." The thalamus and posterior temporal cortex are considered part of the auditory processing pathway (Ramadoss \& Boatman, 2015) and plausibly lesions may have disrupted identification of prosodic features. The two patients with selective deficits in prosody production had right frontal lesions. We did not carry out adequate testing to determine whether their deficits were in access to the affective prosody lexicon (for output) or in motor programming of the speech articulators. The left inferior frontal cortex has been shown to have a role in both motor programming of speech articulation (Fridriksson, Moser, Ryalls, \& Bonilha, 2009; Fridriksson et al., 2008; Hillis et al., 2004), but also access to the output lexicon (Hillis, Wityk, Barker, \& Caramazza, 2003) so in either case, a comparable role of right inferior frontal cortex in affective prosody production would be consistent with the affective prosody network "mirroring" the LH language network. Finally, patients with impairments in both recognition and expression of affective prosody had lesions centering on right anterior temporal pole, indicating this area may be one area critical for the one "shared" component of affective prosody recognition and expression - the semantic representation of emotions (see also Henry et al., 2014 for similar claims).

\section{Directions for Future Research}

Future investigations will require innovative, detailed analysis of performance across tasks to identify patients with selective deficits to the distinct cognitive processes that underlie 
prosody recognition and expression depicted in Figure 4. Future goals include identifying patients with selective impairment in accessing the affective prosody lexicon, with spared auditory analysis and semantic representations of affect; and other patients who show selective impairment in semantic representations of affect, but spared auditory analysis and affective prosody lexicon. Furthermore, larger scale investigations are needed not only to elucidate impairments in cognitive processes underlying prosody, but also to identify lesions associated with the deficits before reorganization or recovery, and chart the course of recovery over time. Finally, it is hoped that a clearer understanding of the neural and cognitive mechanisms underlying affective prosody impairments will provide the basis for selecting patients for specific motor or linguistic treatments that have been shown to have some effectiveness in improving affective prosody after stroke (Leon et al., 2005; Rosenbek et al., 2006; Rothi, Musson, Rosenbek, \& Sapienza, 2008).

\section{References}

Balan A, Gandour J. Effect of sentence length on the production of linguistic stress by left-and righthemisphere-damaged patients. Brain and Language. 1999; 67(2):73-94. [PubMed: 10092343]

Baum SR, Pell MD, Leonard CL, Gordon JK. The ability of right-and left-hemisphere-damaged individuals to produce and interpret prosodic cues marking phrasal boundaries. Language and Speech. 1997; 40(4):313-330. [PubMed: 9692322]

Beatty WW, Orbelo DM, Sorocco KH, Ross ED. Comprehension of affective prosody in multiple sclerosis. Multiple Sclerosis (Houndmills, Basingstoke, England). 2003; 9(2):148-153.

Benton E, Bryan K. Right cerebral hemisphere damage: Incidence of language problems. International Journal of Rehabilitation Research. Internationale Zeitschrift Fur Rehabilitationsforschung. Revue Internationale De Recherches De Readaptation. 1996; 19(1):47-54. [PubMed: 8730543]

Blake ML, Duffy JR, Myers PS, Tompkins CA. Prevalence and patterns of right hemisphere cognitive/ communicative deficits: Retrospective data from an inpatient rehabilitation unit. Aphasiology. 2002; 16(4-6):537-547.

Blonder LX, Bowers D, Heilman KM. The role of the right hemisphere in emotional communication. Brain: A Journal of Neurology. 1991; 114(Pt 3):1115-1127. [PubMed: 2065243]

Blonder LX, Pettigrew LC, Kryscio RJ. Emotion recognition and marital satisfaction in stroke. Journal of Clinical and Experimental Neuropsychology. 2012; 34(6):634-642. [PubMed: 22439916]

Borod, JC. The neuropsychology of emotion. New York: Oxford University Press; 2000.

Bowers D, Bauer RM, Heilman KM. The nonverbal affect lexicon: Theoretical perspectives from neuropsychological studies of affect perception. Neuropsychology. 1993; 7(4):433.doi: 10.1037/0894-4105.7.4.433

Bowers D, Coslett HB, Bauer RM, Speedie LJ, Heilman KM. Comprehension of emotional prosody following unilateral hemispheric lesions: Processing defect versus distraction defect. Neuropsychologia. 1987; 25(2):317-328. [PubMed: 3601038]

Breitenstein C, Daum I, Ackermann H. Emotional processing following cortical and subcortical brain damage: Contribution of the fronto-striatal circuitry. Behavioural Neurology. 1998; 11(1):29-42. [PubMed: 11568400]

Buchanan TW, Lutz K, Mirzazade S, Specht K, Shah NJ, Zilles K, Jancke L. Recognition of emotional prosody and verbal components of spoken language: An fMRI study. Brain Research. Cognitive Brain Research. 2000; 9(3):227-238. S0926641099000609 [pii]. [PubMed: 10808134]

Cancelliere AE, Kertesz A. Lesion localization in acquired deficits of emotional expression and comprehension. Brain and Cognition. 1990; 13(2):133-147. [PubMed: 1697174]

Carton JS, Kessler EA, Pape CL. Nonverbal decoding skills and relationship well-being in adults. Journal of Nonverbal Behavior. 1999; 23(1):91-100.

Cohen MJ, Riccio CA, Flannery AM. Expressive aprosodia following stroke to the right basal ganglia: A case report. Neuropsychology. 1994; 8(2):242. 
Côté H, Payer M, Giroux F, Joanette Y. Towards a description of clinical communication impairment profiles following right-hemisphere damage. Aphasiology. 2007; 21(68):739-749.

Cruttenden, A. Intonation. Cambridge: Cambridge University Press; 1997.

Dara C, Bang J, Gottesman RF, Hillis AE. Right hemisphere dysfunction is better predicted by emotional prosody impairments as compared to neglect. Journal of Neurology \& Translational Neuroscience. 2014; 2(1):1037. [PubMed: 24945016]

Freeman TW, Hart J, Kimbrell T, Ross ED. Comprehension of affective prosody in veterans with chronic posttraumatic stress disorder. The Journal of Neuropsychiatry and Clinical Neurosciences. 2009; 21(1):52-58. DOI: 10.1176/appi.neuropsych.21.1.52 [PubMed: 19359452]

Fridriksson J, Moser D, Ryalls J, Bonilha L. Modulation of frontal lobe speech areas associated with the production and perception of speech movements. Journal of Speech, Language, and Hearing Research. 2009; 52(3):812-819.

Fridriksson J, Moss J, Davis B, Baylis GC, Bonilha L, Rorden C. Motor speech perception modulates the cortical language areas. NeuroImage. 2008; 41(2):605-613. doi:http://dx.doi.org/10.1016/ j.neuroimage.2008.02.046. [PubMed: 18396063]

Gandour J, Baum SR. Production of stress retraction by left-and right-hemisphere-damaged patients. Brain and Language. 2001; 79(3):482-494. [PubMed: 11781055]

Goodglass, H., Kaplan, E. Boston diagnostic aphasia examination. Philadelphia, PA: Lea and Febiger; 1983.

Gorelick PB, Ross ED. The aprosodias: Further functional-anatomical evidence for the organisation of affective language in the right hemisphere. Journal of Neurology, Neurosurgery, and Psychiatry. 1987; 50(5):553-560.

Heilman, KM. Matter of mind: A neurologist's view of brain-behavior relationships. New York: Oxford University Press; 2002.

Heilman KM, Bowers D, Speedie L, Coslett HB. Comprehension of affective and nonaffective prosody. Neurology. 1984; 34(7):917-921. [PubMed: 6539867]

Henry ML, Wilson SM, Ogar JM, Sidhu MS, Rankin KP, Cattaruzza T, ... Seeley WW. Neuropsychological, behavioral, and anatomical evolution in right temporal variant frontotemporal dementia: A longitudinal and post-mortem single case analysis. Neurocase. 2014; 20(1):100-109. [PubMed: 23171151]

Hillis AE, Tippett DC. Stroke recovery: Surprising influences and residual consequences. Advances in Medicine. 2014; 2014

Hillis AE, Wityk RJ, Barker PB, Caramazza A. Neural regions essential for writing verbs. Nature Neuroscience. 2003; 6(1):19-20. [PubMed: 12469127]

Hillis AE, Wityk RJ, Tuffiash E, Beauchamp NJ, Jacobs MA, Barker PB, Selnes OA. Hypoperfusion of wernicke's area predicts severity of semantic deficit in acute stroke. Annals of Neurology. 2001; 50(5):561-566. [PubMed: 11706960]

Hillis AE, Work M, Barker PB, Jacobs MA, Breese EL, Maurer K. Re-examining the brain regions crucial for orchestrating speech articulation. Brain: A Journal of Neurology. 2004; 127(Pt 7):14791487. DOI: 10.1093/brain/awh172 [PubMed: 15090478]

Jackson JH. On the nature of duality of the brain. Brain. 1874/1915; 38:96-103. (Original work published 1874). DOI: 10.1093/brain/38.1-2.96

Karow CM, Connors EC. Affective communication in normal and brain-damaged adults: An overview. Seminars in Speech and Language. 2003; 24(2):69-91. DOI: 10.1055/s-2003-38900 [PubMed: 12709882]

Kreisler A, Godefroy O, Delmaire C, Debachy B, Leclercq M, Pruvo JP, Leys D. The anatomy of aphasia revisited. Neurology. 2000; 54(5):1117-1123. [PubMed: 10720284]

Leon SA, Rosenbek JC, Crucian GP, Hieber B, Holiway B, Rodriguez AD, Gonzalez-Rothi L. Active treatments for aprosodia secondary to right hemisphere stroke. Journal of Rehabilitation Research and Development. 2005; 42(1):93-102. [PubMed: 15742253]

Mah YH, Jager R, Kennard C, Husain M, Nachev P. A new method for automated high-dimensional lesion segmentation evaluated in vascular injury and applied to the human occipital lobe. Cortex; a Journal Devoted to the Study of the Nervous System and Behavior. 2014; 56:51-63. DOI: 10.1016/ j.cortex.2012.12.008 [PubMed: 23347558] 
Nöth E, Batliner A, Kießling A, Kompe R, Niemann H. Verbmobil: The use of prosody in the linguistic components of a speech understanding system. Speech and Audio Processing, IEEE Transactions On. 2000; 8(5):519-532.

Orbelo DM, Grim MA, Talbott RE, Ross ED. Impaired comprehension of affective prosody in elderly subjects is not predicted by age-related hearing loss or age-related cognitive decline. Journal of Geriatric Psychiatry and Neurology. 2005; 18(1):25-32. 18/1/25 [pii]. [PubMed: 15681625]

Pell MD. Cerebral mechanisms for understanding emotional prosody in speech. Brain and Language. 2006; 96(2):221-234. [PubMed: 15913754]

Pihan H, Altenmuller E, Hertrich I, Ackermann H. Cortical activation patterns of affective speech processing depend on concurrent demands on the subvocal rehearsal system. A DC-potential study. Brain: A Journal of Neurology. 2000; 123(Pt 11):2338-2349. [PubMed: 11050033]

Pobric G, Jefferies E, Lambon Ralph MA. Amodal semantic representations depend on both anterior temporal lobes: Evidence from repetitive transcranial magnetic stimulation. Neuropsychologia. 2010; 48(5):1336-1342. doi:http://dx.doi.org/10.1016/j.neuropsychologia.2009.12.036. [PubMed: 20038436]

Praat. Praat: doing phonetics by computer. n.d. Retrieved from http://www.fon.hum.uva.nl/praat/

Ramadoss, D., Boatman, D. Neurobiological bases of auditory processing. In: Hillis, AE., editor. The Handbook of Adult Language Disorders. 2. New York: Psychology Press; 2015.

Robin DA, Tranel D, Damasio H. Auditory perception of temporal and spectral events in patients with focal left and right cerebral lesions. Brain and Language. 1990; 39(4):539-555. [PubMed: 2076495]

Rorden C, Karnath H. Using human brain lesions to infer function: A relic from a past era in the fMRI age? Nature Reviews Neuroscience. 2004; 5(10):812-819.

Rosenbek JC, Rodriguez AD, Hieber B, Leon SA, Crucian GP, Ketterson TU, ... Rothi LJG. Effects of two treatments for aprosodia secondary to acquired brain injury. Journal of Rehabilitation Research and Development. 2006; 43(3):379. [PubMed: 17041823]

Ross E. Right hemisphere syndromes and the neurology of emotion. Behavioral Neurology and the Legacy of Norman Geschwind. 1997:183-191.

Ross, ED. Affective prosody and the aprosodias. In: Mesulam, MM., editor. Principles of Behavioral and Cognitive Neurology. New York: Oxford University Press; 2000. p. 316-331.

Ross ED. Cerebral localization of functions and the neurology of language: Fact versus fiction or is it something else? The Neuroscientist: A Review Journal Bringing Neurobiology, Neurology and Psychiatry. 2010; 16(3):222-243. DOI: 10.1177/1073858409349899

Ross ED, Mesulam M. Dominant language functions of the right hemisphere?: Prosody and emotional gesturing. Archives of Neurology. 1979; 36(3):144-148. [PubMed: 435134]

Ross ED, Monnot M. Neurology of affective prosody and its functional-anatomic organization in right hemisphere. Brain and Language. 2008; 104(1):51-74. doi:http://dx.doi.org.proxy1.library.jhu.edu/ 10.1016/j.bandl.2007.04.007. [PubMed: 17537499]

Ross ED, Orbelo DM, Cartwright J, Hansel S, Burgard M, Testa JA, Buck R. Affective-prosodic deficits in schizophrenia: Comparison to patients with brain damage and relation to schizophrenic symptoms [corrected]. Journal of Neurology, Neurosurgery, and Psychiatry. 2001; 70(5):597-604.

Ross ED, Shayya L, Rousseau JF. Prosodic stress: Acoustic, aphasic, aprosodic and neuroanatomic interactions. Journal of Neurolinguistics. 2013; 26(5):526-551.

Ross ED, Thompson RD, Yenkosky J. Lateralization of affective prosody in brain and the callosal integration of hemispheric language functions. Brain and Language. 1997; 56(1):27-54. [PubMed: 8994697]

Roth DL, Haley WE, Clay OJ, Perkins M, Grant JS, Rhodes JD, ... Howard G. Race and gender differences in 1-year outcomes for community-dwelling stroke survivors with family caregivers. Stroke. 2011; 42(3):626-631. DOI: 10.1161/STROKEAHA.110.595322 [PubMed: 21257820]

Rothi LJG, Musson N, Rosenbek JC, Sapienza CM. Neuroplasticity and rehabilitation research for speech, language, and swallowing disorders. Journal of Speech, Language, and Hearing Research. 2008; 51(1):S222-S224. 
Samuelsson H, Jensen C, Ekholm S, Naver H, Blomstrand C. Anatomical and neurological correlates of acute and chronic visuospatial neglect following right hemisphere stroke. Cortex. 1997; 33(2): 271-285. [PubMed: 9220258]

Schirmer A, Alter K, Kotz SA, Friederici AD. Lateralization of prosody during language production: A lesion study. Brain and Language. 2001; 76(1):1-17. doi: http://dx.doi.org/10.1006/brln. 2000.2381. [PubMed: 11161351]

Shah AP, Baum SR, Dwivedi VD. Neural substrates of linguistic prosody: Evidence from syntactic disambiguation in the productions of brain-damaged patients. Brain and Language. 2006; 96(1): 78-89. [PubMed: 15922444]

Sidtis DVL, Pachana N, Cummings JL, Sidtis JJ. Dysprosodic speech following basal ganglia insult: Toward a conceptual framework for the study of the cerebral representation of prosody. Brain and Language. 2006; 97(2):135-153. [PubMed: 16271755]

Testa JA, Beatty WW, Gleason AC, Orbelo DM, Ross ED. Impaired affective prosody in AD: Relationship to aphasic deficits and emotional behaviors. Neurology. 2001; 57(8):1474-1481. [PubMed: 11673592]

Trauner DA, Ballantyne A, Friedland S, Chase C. Disorders of affective and linguistic prosody in children after early unilateral brain damage. Annals of Neurology. 1996; 39(3):361-367. [PubMed: 8602755]

Tucker DM, Watson RT, Heilman KM. Discrimination and evocation of affectively intoned speech in patients with right parietal disease. Neurology. 1977; 27(10):947-950. [PubMed: 561908]

Vallar G. Extrapersonal visual unilateral spatial neglect and its neuroanatomy. NeuroImage. 2001; 14(1):S52-S58. [PubMed: 11373133]

Van Lancker D. Cerebral lateralization of pitch cues in the linguistic signal. Research on Language \& Social Interaction. 1980; 13(2):201-277.

Van Lancker D, Sidtis JJ. The identification of affective-prosodic stimuli by left-and right-hemispheredamaged SubjectsAll errors are not created equal. Journal of Speech, Language, and Hearing Research. 1992; 35(5):963-970.

Voeller KK. Clinical neurologic aspects of the right-hemisphere deficit syndrome. Journal of Child Neurology. 1995; 10(1 Suppl):S16-S22. [PubMed: 7751549]

Walker JP, Joseph L, Goodman J. The production of linguistic prosody in subjects with aphasia. Clinical Linguistics \& Phonetics. 2009; 23(7):529-549. [PubMed: 19585312]

Walker JP, Pelletier R, Reif L. The production of linguistic prosodic structures in subjects with right hemisphere damage. Clinical Linguistics \& Phonetics. 2004; 18(2):85-106. [PubMed: 15086132]

Weintraub S, Mesulam M. Developmental learning disabilities of the right hemisphere: Emotional, interpersonal, and cognitive components. Archives of Neurology. 1983; 40(8):463-468. [PubMed: 6870605]

Weintraub S, Mesulam M, Kramer L. Disturbances in prosody: A right-hemisphere contribution to language. Archives of Neurology. 1981; 38(12):742-744. [PubMed: 7316838]

Wertz RT, Henschel CR, Auther LL, Ashford JR, Kirshner HS. Affective prosodic disturbance subsequent to right hemisphere stroke: A clinical application. Journal of Neurolinguistics. 1998; 11(1):89-102.

Wolfe GI, Ross ED. Sensory aprosodia with left hemiparesis from subcortical infarction: Right hemisphere analogue of sensory-type aphasia with right hemiparesis? Archives of Neurology. 1987; 44(6):668-671. [PubMed: 3579688]

Wong PC. Hemispheric specialization of linguistic pitch patterns. Brain Research Bulletin. 2002; 59(2):83-95. [PubMed: 12379438]

Wymer JH, Lindman LS, Booksh RL. A neuropsychological perspective of aprosody: Features, function, assessment, and treatment. Applied Neuropsychology. 2002; 9(1):37-47. [PubMed: 12173749]

Zahn R, Moll J, Iyengar V, Huey ED, Tierney M, Krueger F, Grafman J. Social conceptual impairments in frontotemporal lobar degeneration with right anterior temporal hypometabolism. Brain: A Journal of Neurology. 2009; 132(Pt 3):604-616. DOI: 10.1093/brain/awn343 [PubMed: 19153155] 


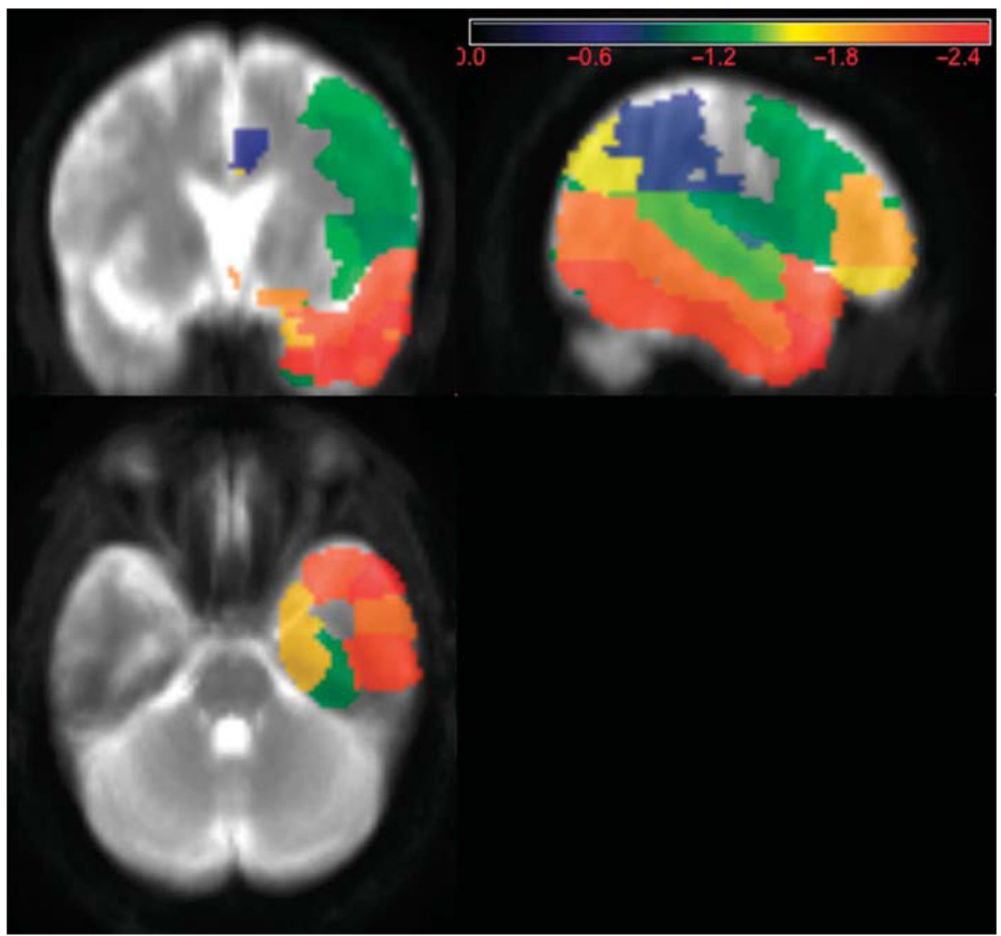

Figure 1. Lesion Map of Patients With Expressive Prosody Impairments

Note. Regions most strongly associated with severity of expressive prosody deficit (measured by mean $\mathrm{F}_{0}-\mathrm{CV} \%$ ), in 9 patients, of whom the majority had deficits in both expression and recognition of affective prosody. This figure is shown in anatomical orientation (right hemisphere on the right). 


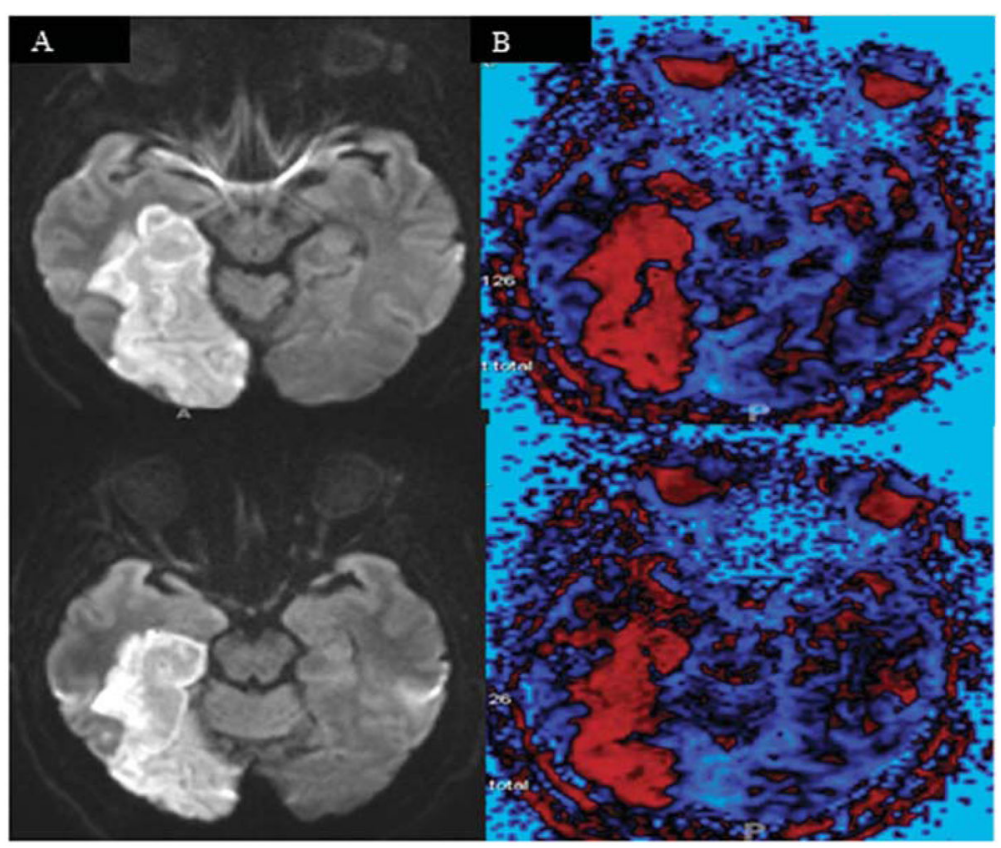

Figure 2. MRI of the Single Patient With an Abnormally Low $\mathbf{F}_{0}-\mathrm{CV} \%$ in the Picture Description Task

Note. MR Diffusion Weight Image (left) and Perfusion Weight Imaging (right) showing hypoperfusion (in red) in the inferior temporal cortex, including left temporal pole, in a patient with impaired affective prosody expression and production. This figure is oriented according to radiological convention (right hemisphere on the left). 


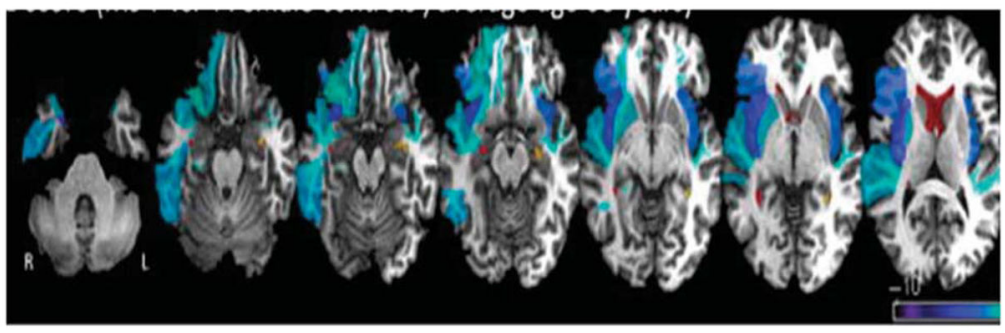

Figure 3. Z-Score of Atrophy of byFTD Patient as Compared to Age-Matched Controls Note. Areas of greatest atrophy compared with age-matched healthy controls in a patient with severe deficits in affective prosody expression and recognition due to behavioral variant frontotemporal dementia (byFTD). Voxels in blue are voxels that are smaller in the byFTD patient; voxels in red are voxels that are larger in the byFTD (the ventricles). This figure is oriented according to radiological convention (right hemisphere on the left). 


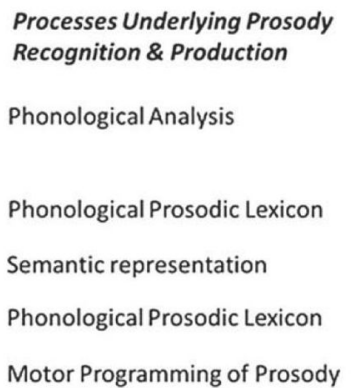

Motor Programming of Prosody

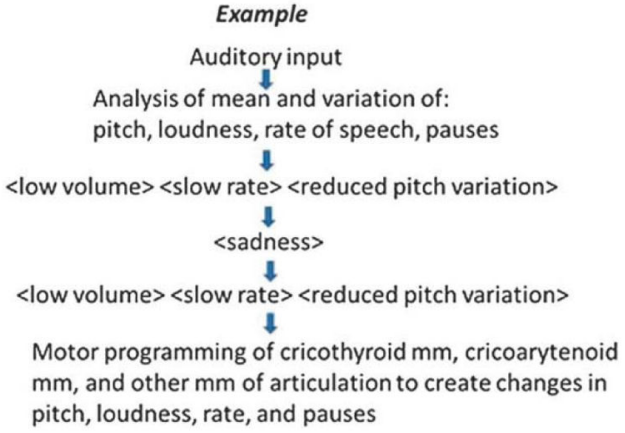

Figure 4.

A Schematic Representation of the Cognitive Mechanisms Underlying Affective Prosody Recognition and Production. 